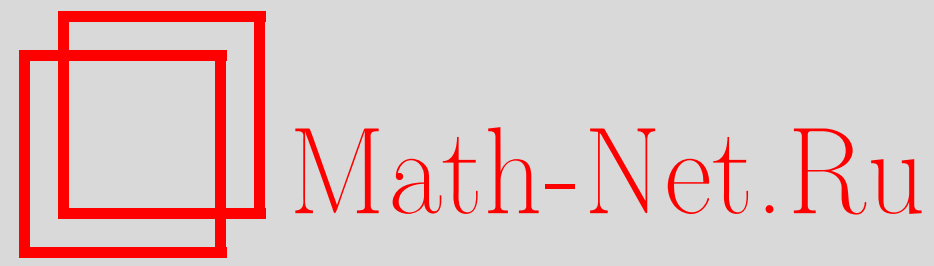

А. Г. Елисеев, Т. А. Ратникова, Д. А. Шапошникова, Задача инициализации сингулярно возмущенных интегро-дифференциальных и интегральных уравнений Вольтерра второго рода, Итоги науки и техн. Сер. Соврем. мат. и ее прил. Tемат. обз., 2021, том 191, 38-46

DOI: https://doi.org/10.36535/0233-6723-2021-191-38-46

Использование Общероссийского математического портала Math-Net.Ru подразумевает, что вы прочитали и согласны с пользовательским соглашением

http://www.mathnet.ru/rus/agreement

Параметры загрузки:

IP : 54.172 .240 .79

26 апреля 2023 г., 10:37:22 
ИТОГИ НАУКИ И ТЕХНИКИ.

Современная математика и ее приложения.

Тематические обзоры.

Том 191 (2021). С. $38-46$

DOI: 10.36535/0233-6723-2021-191-38-46

УДК 517.95

\title{
ЗАДАЧА ИНИЦИАЛИЗАЦИИ СИНГУЛЯРНО ВОЗМУЩЕННЫХ ИНТЕГРО-ДИФФЕРЕНЦИАЛЬНЫХ И ИНТЕГРАЛЬНЫХ УРАВНЕНИЙ ВОЛЬТЕРРА ВТОРОГО РОДА
}

\author{
(c) 2021 г. $\quad$ А. Г. ЕЛИСЕЕВ, Т. А. РАТНИКОВА, Д. А. ШАПОШНИКОВА
}

АннотАция. В статье при помощи метода регуляризации С. А. Ломова решена проблема инициализации для сингулярно возмущенных интегро-дифференциальных и интегральных систем уравнений Вольтерра второго рода.

Ключевые слова: сингулярное возмущение, задача инициализации, метод регуляризации, интегро-дифференциальное уравнение Вольтерра.

\section{INITIALIZATION PROBLEM FOR SINGULARLY PERTURBED INTEGRO-DIFFERENTIAL AND INTEGRAL VOLTERRA EQUATIONS OF THE SECOND KIND}

\author{
(c) 2021 A. G. ELISEEV, T. A. RATNIKOVA, D. A. SHAPOSHNIKOVA
}

\begin{abstract}
The initialization problem for singularly perturbed integro-differential and integral systems of Volterra equations of the second kind is solved by the Lomov regularization method.

Keywords and phrases: singular perturbation, initialization problem, regularization method, Volterra integro-differential equation.
\end{abstract}

AMS Subject Classification: 34K26, 34E20

Задаче инициализации, возникшей первоначально в метрологии, посвящена обширная литература (см. $[1,3,4])$.

При малых числах Россби решение атмосферных моделей зависит от двух временных масштабов: «медленного» времени и «быстрого» времени $t / \varepsilon$, где $\varepsilon$ - число Россби. Быстро осциллирующие члены в решении несущественны для прогноза погоды на больших интервалах времени. Поэтому возникает потребность в специальной процедуре - инициализации, которая подавляла бы быстро осциллирующие или быстро растущие волны масштабных разложений. В [4] доказана разрешимость задачи инициализации для двухслойной квазигеострофической модели общей циркуляции атмосферы. Построена полуявная спектрально-разностная схема.

В данной статье задача инициализации рассматривается с позиции метода регуляризации С. А. Ломова (см. [2]) для сингулярно возмущенных интегро-дифференциальных и интегральных систем уравнений Вольтерра второго рода. 


\section{1. Спектр предельного оператора чисто мнимый.}

1.1. Задача Коши для интегро-дифференииальной системы. Пусть дана задача Коши для интегро-дифференциальной системы:

$$
\left\{\begin{array}{l}
\varepsilon \dot{u}(t, \varepsilon)+A(t) u(t, \varepsilon)+\int_{0}^{t} K(t, s) u(s, \varepsilon) d s=h(t), \\
u(0, \varepsilon)=u^{0}
\end{array}\right.
$$

при следующих условиях:

$$
\begin{gathered}
h(t) \in C^{\infty}([0, T]) ; \quad A(t) \in C^{\infty}([0, T]) ; \\
\operatorname{Sp}(A(t))=\{ \pm i \lambda(t)\}, \quad \lambda(t)>0 ; \\
K(t, s) \in C^{\infty} \quad(0 \leqslant s \leqslant t \leqslant T) ; \\
A(t)=\lambda_{1}(t) P_{1}(t)+\lambda_{2}(t) P_{2}(t), \quad P_{1}(t)+P_{2}(t)=I, \quad \lambda_{1}(t)=i \lambda(t), \quad \lambda_{2}(t)=-i \lambda(t) .
\end{gathered}
$$

Согласно методу регуляризации решение (1) имеет вид:

$$
u(t, \varepsilon)=e^{\frac{i}{\varepsilon} \varphi(t)} x(t, \varepsilon)+e^{-\frac{i}{\varepsilon} \varphi(t)} y(t, \varepsilon)+z(t, \varepsilon),
$$

где

$$
\varphi(t)=\int_{0}^{t} \lambda(s) d s,
$$

$x(t, \varepsilon), y(t, \varepsilon), z(t, \varepsilon) \in C^{\infty}[0, T]$ - регулярные по $\varepsilon$ степенные ряды.

Для решения задачи инициализации надо подобрать такие начальные условия $u^{0}(\varepsilon)$, чтобы быстро осциллирующие составляющие решения $x(t, \varepsilon), y(t, \varepsilon)$ обратились в ноль или их влияние было минимизировано.

Приведем алгоритм решения задачи. Будем искать решение в виде

$$
u(t, \varepsilon)=\sum_{k=0}^{n} \varepsilon^{k}\left(e^{\frac{i}{\varepsilon} \varphi(t)} x_{k}(t)+e^{-\frac{i}{\varepsilon} \varphi(t)} y_{k}(t)+z_{k}(t)\right)+\varepsilon^{n+1} R_{n}(t, \varepsilon) .
$$

Подставив (4) в систему (1), получим серию итерационных задач для определения $x_{k}(t), y_{k}(t)$, $z_{k}(t)$ и задачу для определения остатка $R_{n}(t, \varepsilon)$.

Выпишем две итерационные задачи:

$$
\begin{aligned}
& \left\{\begin{array}{l}
\left(A(t)-\lambda_{1}(t) I\right) x_{0}(t)=0, \\
\left(A(t)-\lambda_{2}(t) I\right) y_{0}(t)=0, \\
t \\
A(t) z_{0}(t)+\int_{0}^{t} K(t, s) z_{0}(s) d s=h(t), \\
x_{0}(0)+y_{0}(0)+z_{0}(0)=u_{0}^{0}
\end{array}\right. \\
& \left\{\begin{array}{l}
\left(A(t)-\lambda_{1}(t) I\right) x_{1}(t)=-\dot{x}_{0}(t)-\frac{K(t, t)}{\lambda_{1}(t)} x_{0}(t), \\
\left(A(t)-\lambda_{2}(t) I\right) y_{1}(t)=-\dot{y}_{0}(t)-\frac{K(t, t)}{\lambda_{2}(t)} y_{0}(t), \\
A(t) z_{1}(t)+\int_{0}^{t} K(t, s) z_{1}(s) d s=-\dot{z}_{0}(t)+\frac{K(t, 0)}{\lambda_{1}(0)} x_{0}(0)+\frac{K(t, 0)}{\lambda_{2}(0)} y_{0}(0), \\
x_{1}(0)+y_{1}(0)+z_{1}(0)=u_{1}^{0} .
\end{array}\right.
\end{aligned}
$$


Решение (5) имеет вид:

$$
\begin{gathered}
x_{0}(t)=P_{1}(t) x_{0}(t), \quad y_{0}(t)=P_{2}(t) y_{0}(t) \\
z_{0}(t)=\left(I+\int_{0}^{t} A^{-1}(t) K(t, s) \bullet d s\right)^{-1} A^{-1}(t) h(t)=D(t) H(t) .
\end{gathered}
$$

Здесь

$$
D(t)=\left(I+\int_{0}^{t} A^{-1}(t) K(t, s) \bullet d s\right)^{-1} A^{-1}(t) \frac{d}{d t}, \quad H(t)=\int_{0}^{t} h(s) d s .
$$

Если выбрать в системе (5) начальное условие $u_{0}^{0}=z_{0}(0)=\left.D(t) H(t)\right|_{t=0}$, то $x_{0}(0)+y_{0}(0)=0$. Произвольные собственные векторы $x_{0}(t), y_{0}(t)$ определяются из условия разрешимости системы (6):

$$
\begin{aligned}
& \left\{\begin{array}{l}
\frac{d}{d t}\left(P_{1}(t) x_{0}(t)\right)=\left(\dot{P}_{1}(t)-\frac{1}{\lambda_{1}(t)} P_{1}(t) K(t, t)\right) P_{1}(t) x_{0}(t)=0, \\
P_{1}(0) x_{0}(0)=0 ;
\end{array}\right. \\
& \left\{\begin{array}{l}
\frac{d}{d t}\left(P_{2}(t) y_{0}(t)\right)=\left(\dot{P}_{2}(t)-\frac{1}{\lambda_{2}(t)} P_{2}(t) K(t, t)\right) P_{2}(t) y_{0}(t)=0, \\
P_{2}(0) y_{0}(0)=0 .
\end{array}\right.
\end{aligned}
$$

Следовательно, $P_{1}(t) x_{0}(t) \equiv 0, P_{2}(t) y_{0}(t) \equiv 0$. Начальные условия нулевые, так как мы выбрали

$$
u_{0}^{0}=\left.D(t) H(t)\right|_{t=0}=A^{-1}(0) h(0) .
$$

Итерационная задача (6) относительно $x_{1}(t), y_{1}(t)$ будет иметь вид (5). При этом

$$
z_{1}(t)=-D^{2}(t) H(t), \quad u_{1}^{0}=-\left.D^{2}(t) H(t)\right|_{t=0} .
$$

Тогда $x_{1}(t) \equiv 0, y_{1}(t) \equiv 0$. Продолжая этот процесс, получим формальное решение задачи инициализации

$$
u(t, \varepsilon)=\sum_{k=0}^{n}(-1)^{k} D^{(k+1)}(t) H(t)+\varepsilon^{n+1} R_{n}(t, \varepsilon) .
$$

Заметим, что сумма не содержит быстро осциллирующих слагаемых. Если взять в качестве начального условия

$$
u^{0}(\varepsilon)=\left.\sum_{k=0}^{\infty}(-1)^{k} \varepsilon^{k} D^{(k+1)}(t) H(t)\right|_{t=0},
$$

то получим решение системы (1):

$$
u(t, \varepsilon)=\sum_{k=0}^{\infty}(-1)^{k} \varepsilon^{k} D^{(k+1)}(t) H(t) .
$$

Докажем асимптотичность полученного ряда, оценив остаток $R_{n}(t, \varepsilon)$ и тем самым оценив амплитуду быстрых осцилляций. Пусть

$$
u(t, \varepsilon)=\sum_{k=0}^{n}(-1)^{k} \varepsilon^{k} D^{(k+1)}(t) H(t)+\varepsilon^{n+1} R_{n+1}(t, \varepsilon) .
$$

Задача для определения $R_{n}(t, \varepsilon)$ имеет вид

$$
\left\{\begin{array}{l}
\varepsilon \dot{R}_{n+1}(t, \varepsilon)+A(t) R_{n+1}(t, \varepsilon)+\int_{0}^{t} K(t, s) R_{n+1}(s, \varepsilon) d s=(-1)^{n+1} \frac{d}{d t} D^{(n+1)}(t) H(t), \\
R_{n+1}(0, \varepsilon)=0 .
\end{array}\right.
$$

Несложно показать, что верна следующая теорема. 
Теорема 1. Рассмотрим на отрезке $[0, T]$ задачу (1) с начальным условием

$$
u^{0}(\varepsilon)=\left.\sum_{k=0}^{\infty}(-1)^{k} \varepsilon^{k} D^{(k+1)}(t) H(t)\right|_{t=0}
$$

и предположим, что выполнены условия (2). Тогда имеет место оценка

$$
\left\|u(t, \varepsilon)-\sum_{k=0}^{n}(-1)^{k} \varepsilon^{k} D^{(k+1)}(t) H(t)\right\|_{C[0, T]}=O\left(\varepsilon^{n+1}\right) .
$$

Как следствие теоремы, амплитуда быстро осциллирующих слагаемых уменьшена до $O\left(\varepsilon^{n+1}\right)$. Учитывая, что спектр оператора чисто мнимый, легко показать, что $\left\|R_{n+1}\right\|_{C[0, T]} \leqslant M$. Таким образом, амплитуда быстро осциллирующих решений не превышает $M \varepsilon^{n+1}$. Используя такой подход, можно уменьшать влияние быстро осциллирующих слагаемых на решение интегро-дифференциальных систем Вольтерра второго рода.

1.2. Интегральная система Вольтерра второго рода. Интегральная система Вольтерра второго рода с чисто мнимым спектром предельного оператора

$$
\varepsilon u(t, \varepsilon)+\int_{0}^{t} K(t, s) u(s, \varepsilon) d s=h(t),
$$

сводится к эквивалентной интегро-дифференциальной системе

$$
\left\{\begin{array}{l}
\varepsilon \dot{u}(t, \varepsilon)+A(t) u(t, \varepsilon)+\int_{0}^{t} \dot{K}(t, s) u(s, \varepsilon) d s=\dot{h}(t), \\
u(0, \varepsilon)=\frac{h(0)}{\varepsilon}
\end{array}\right.
$$

где $A(t) \equiv K(t, t)$.

Пусть выполнены условия (2) для задачи (10). Специфика задачи (10) состоит в том, что начальные условия диктуются правой частью (9). Для того чтобы убрать быстро осциллирующие слагаемые в решении (10), поставим задачу Коши:

$$
\left\{\begin{array}{l}
\varepsilon \dot{u}(t, \varepsilon)+A(t) u(t, \varepsilon)+\int_{0}^{t} \dot{K}(t, s) u(s, \varepsilon) d s=\dot{h}(t), \\
u(0, \varepsilon)=\frac{h(0)}{\varepsilon}+u^{0}(\varepsilon)
\end{array}\right.
$$

где

Здесь

$$
u^{0}(\varepsilon)=-\frac{h(0)}{\varepsilon}+\left.\sum_{k=0}^{\infty}(-1)^{k} \varepsilon^{k} D^{(k+1)}(t) h(t)\right|_{t=0} .
$$

$$
D(t)=\left(I+\int_{0}^{t} A^{-1}(t) K(t, s) \bullet d s\right)^{-1} A^{-1}(t) \frac{d}{d t} .
$$

Тогда формальное решение (11) запишется в виде

$$
u(t, \varepsilon)=\sum_{k=0}^{\infty}(-1)^{k} \varepsilon^{k} D^{(k+1)}(t) h(t) .
$$

Асимптотичность ряда (12) доказывается аналогично.

Замечание. Если $\left.D^{(k+1)}(t) h(t)\right|_{t=0}=0$ для всех $k=-1,0,1, \ldots$, то решение (10) автоматически не будет содержать быстро осциллирующих компонент. 
2. Спектр предельного оператора содержит положительное собственное значение. Специфику решения задачи инициализации в этом случае можно прояснить на скалярном интегро-дифференциальном и интегральном уравнениях Вольтерра второго рода. Пусть дана задача Коши

при условиях

$$
\left\{\begin{array}{l}
\varepsilon \dot{u}(t, \varepsilon)-\lambda(t) u(t, \varepsilon)+\int_{0}^{t} K(t, s) u(s, \varepsilon) d s=h(t), \\
u(0, \varepsilon)=u^{0}(\varepsilon) .
\end{array}\right.
$$

$$
\begin{gathered}
h(t) \in C^{\infty}[0, T] ; \quad K(t, s) \in C^{\infty} \quad(0 \leqslant s \leqslant t \leqslant T) ; \\
\lambda(t) \in C^{\infty}[0, T] ; \quad \lambda(t)>0, \quad t \in[0, T] ;
\end{gathered}
$$

$u^{0}(\varepsilon)$ подлежит определению.

Решение (13) по методу регуляризации запишется в виде

$$
u(t, \varepsilon)=\exp \left(\frac{\varphi(t)}{\varepsilon}\right) x(t, \varepsilon)+y(t, \varepsilon), \quad \text { где } \quad \varphi(t)=\int_{0}^{t} \lambda(s) d s
$$

$x(t, \varepsilon), y(t, \varepsilon) \in C^{\infty}[0, T] ; x(t, \varepsilon), y(t, \varepsilon)$ - регулярные по $\varepsilon$ степенные ряды. Для решения задачи инициализации необходимо подобрать $u^{0}(\varepsilon)$ таким образом, чтобы $x(t, \varepsilon) \equiv 0$.

Если положить

$$
u^{0}(\varepsilon)=-\left.\sum_{k=0}^{\infty} \varepsilon^{k} D^{(k+1)}(t) H(t)\right|_{t=0}, \quad \text { где } \quad H(t)=\int_{0}^{t} h(s) d s,
$$

то формальное решение (13) запишется в виде

$$
u(t, \varepsilon)=-\sum_{k=0}^{\infty} \varepsilon^{k} D^{(k+1)}(t) H(t) .
$$

где

$$
D(t)=\left(1-\int_{0}^{t} \frac{1}{\lambda(t)} K(t, s) \bullet d s\right)^{-1} \frac{1}{\lambda(t)} \frac{d}{d t} .
$$

Существенное отличие от случая с чисто мнимым спектром состоит в том, что здесь необходимо найти весь ряд $u^{0}(\varepsilon)$ и найти условия для $h(t)$, при которых ряд $(15)$ сходится. В противном случае нельзя провести оценку остатка при доказательстве асимптотичности ряда (15) при $\varepsilon \rightarrow 0$.

Добавим к условиям (14) условие на правую часть, а именно:

$$
\exists \nu>0 \exists C>0 \forall t \in[0, T] \quad\left|D^{k}(t) H(t)\right| \leqslant C \nu^{k}, \quad k=0,1,2, \ldots
$$

Тогда ряд (15) сходится равномерно, а следовательно, является асимптотическим.

Рассмотрим случай интегрального уравнения Вольтерра второго рода

$$
\varepsilon u(t, \varepsilon)+\int_{0}^{t} K(t, s) u(s, \varepsilon) d s=h(t) .
$$

Сводим (17) к эквивалентной интегро-дифференциальной задаче Коши:

$$
\left\{\begin{array}{l}
\varepsilon \dot{u}(t, \varepsilon)-\lambda(t) u(t, \varepsilon)+\int_{0}^{t} \dot{K}(t, s) u(s, \varepsilon) d s=\dot{h}(t), \\
u(0, \varepsilon)=\frac{h(0)}{\varepsilon}
\end{array}\right.
$$


где $\lambda(t) \equiv-K(t, t)$. Предполагается, что для (18) выполнены условия (14). Условие (16) заменяется на

$$
\exists \nu>0 \exists C>0 \forall t \in[0, T] \quad\left|D^{k}(t) h(t)\right| \leqslant C \nu^{k}, \quad k=0,1,2, \ldots,
$$

где

Тогда задача Коши

$$
D(t)=\left(1-\int_{0}^{t} \frac{1}{\lambda(t)} \dot{K}(t, s) \bullet d s\right)^{-1} \frac{1}{\lambda(t)} \frac{d}{d t} .
$$

где

$$
\left\{\begin{array}{l}
\varepsilon \dot{u}(t, \varepsilon)-\lambda(t) u(t, \varepsilon)+\int_{0}^{t} \dot{K}(t, s) u(s, \varepsilon) d s=\dot{h}(t) \\
u(0, \varepsilon)=u^{0}(\varepsilon)
\end{array}\right.
$$

$$
u^{0}(\varepsilon)=-\frac{h(0)}{\varepsilon}-\left.\sum_{k=0}^{\infty} \varepsilon^{k} D^{(k+1)}(t) h(t)\right|_{t=0},
$$

в своем решении не содержит экспоненциально растущих слагаемых.

Решение (19) примет вид

$$
u(t, \varepsilon)=-\sum_{k=0}^{\infty} \varepsilon^{k} D^{(k+1)}(t) h(t) .
$$

Замечание. Если выбрать $h(t)$ из условия, что $D^{n}(t) h(t) \equiv 0$, то решение получится в виде конечной суммы:

$$
u(t, \varepsilon)=-\sum_{k=0}^{n-1} \varepsilon^{k} D^{k}(t) h(t) .
$$

Как отмечалось выше, в случае $\lambda(t)>0$ для решения задачи инициализации необходимо и достаточно выполнение условий

$$
u^{0}(\varepsilon)=-\left.\sum_{k=0}^{\infty} \varepsilon^{k} D^{(k+1)}(t) H(t)\right|_{t=0}
$$

для задачи (13) или

$$
u^{0}(\varepsilon)=-\left.\sum_{k=0}^{\infty} \varepsilon^{k} D^{(k+1)}(t) h(t)\right|_{t=0}-\frac{h(0)}{\varepsilon}
$$

для уравнения (17).

Возникает вопрос: как работать с таким решением? Для этого оценим остаток. Подставим

$$
u(t, \varepsilon)=-\sum_{k=0}^{n} \varepsilon^{k} D^{k}(t) h(t)+\varepsilon^{n+1} R_{n+1}(t, \varepsilon)
$$

в задачу (19) и получим

$$
\left\{\begin{array}{l}
\varepsilon \dot{R}_{n+1}(t, \varepsilon)-\lambda(t) R_{n+1}(t, \varepsilon)+\int_{0}^{t} \dot{K}(t, s) R_{n+1}(s, \varepsilon) d s=-\frac{d}{d t} D^{n}(t) h(t), \\
R_{n+1}(0, \varepsilon)=0
\end{array}\right.
$$

Пусть

$$
R_{n+1}(t, \varepsilon)=\exp \left(\frac{1}{\varepsilon} \int_{0}^{t} \lambda(s) d s\right) r_{n}(t, \varepsilon) .
$$

Тогда 


$$
\begin{aligned}
\varepsilon \dot{r}_{n}+\exp \left(-\frac{1}{\varepsilon} \int_{0}^{t} \lambda(s) d s\right) \int_{0}^{t} \dot{K}(t, s) \exp \left(\int_{0}^{s} \lambda\left(s_{1}\right) d s_{1}\right) & r_{n}(s) d s= \\
& =-\exp \left(-\frac{1}{\varepsilon} \int_{0}^{t} \lambda(s) d s\right) \frac{d}{d t} D^{n}(t) h(t) .
\end{aligned}
$$

Проинтегрируем по $t$ и изменим порядок интегрирования:

$$
\begin{aligned}
\varepsilon r_{n}=\varepsilon r_{n}(0)-\int_{0}^{t} \int_{s}^{t} \dot{K}\left(s_{1}, s\right) \exp \left(-\frac{1}{\varepsilon} \int_{s}^{s_{1}} \lambda\left(s_{2}\right) d s_{2}\right) d s_{1} r_{n}(s) d s- & \\
& -\int_{0}^{t} \exp \left(-\frac{1}{\varepsilon} \int_{0}^{s} \lambda\left(s_{1}\right) d s_{1}\right) \frac{d}{d s} D^{n}(s) h(s) d s .
\end{aligned}
$$

Учитывая, что $\lambda(t) \geqslant \delta>0$, оценим $(21)$ по норме $\mathbb{C}[0, T]$. В результате получим, что $\left\|r_{n}\right\| \leqslant C$. Отсюда

$$
\left\|R_{n}\right\| \leqslant C \exp \left(\frac{1}{\varepsilon} \int_{0}^{t} \lambda(s) d s\right)
$$

Учитывая, что $\delta \leqslant \lambda(t) \leqslant M$ на $[0, T]$, получим

$$
\left\|R_{n}\right\| \leqslant C e^{M T / \varepsilon} .
$$

Тогда

$$
\varepsilon^{n+1}\left\|R_{n}\right\| \leqslant \varepsilon^{n+1} C e^{M T / \varepsilon} .
$$

Минимум оценки, равный $\left(\frac{e M T}{n+1}\right)^{n+1}$, достигается при $\varepsilon^{*}=\frac{M T}{n+1}$. Задав точность $\delta$, т.е. положив $\left(\frac{e M T}{n+1}\right)^{n+1}=\delta$, получим

$$
\varepsilon=\frac{1}{e} \sqrt[n+1]{\delta}
$$

либо, задав $\varepsilon$, подбираем

$$
n=\left[\frac{e M T}{\varepsilon}-1\right]
$$

В заключение рассмотрим, каким условиям должна удовлетворять $h(t)$, чтобы задача инициализации решалась без подбора начального условия $u^{0}(\varepsilon)$. Введем оператор

$$
B(t)=\lambda(t)-\int_{0}^{t} K(t, s) \bullet d s
$$

Теорема 2. Пусть $h(t)$ удовлетворлет следуюшим условиям:

(1) функиия $h(t)$ является В-аналитичной для всех $t \in[0, T]$, за исключением точки $t=0$;

(2) $\left.D^{k}(t) h(t)\right|_{t=0}=0$ для всех $k=0,1,2, \ldots$;

(3) для всех $k=0,1,2, \ldots$ выполнена обратная теорема Абеля в точке $t=0$ :

$$
\lim _{s \rightarrow 0+} \sum_{k=0}^{\infty}\left(\int_{t}^{s} B\left(s_{1}\right) d s_{1}\right)^{k} D^{k}(t) h(t)=\sum_{k=0}^{\infty}\left(\int_{t}^{0} B\left(s_{1}\right) d s_{1}\right)^{k} D^{k}(t) h(t)=0 .
$$


Тогда ряд

$$
u(t, \varepsilon)=-\sum_{k=0}^{\infty} \varepsilon^{k} D^{(k+1)} h(t)
$$

является решением задачи (17).

Доказательство. Разложим $B$-аналитичную функцию $h(t)$ в ряд

$$
h(s)=\sum_{k=0}^{\infty}\left(\int_{t}^{s} B\left(s_{1}\right) d s_{1}\right)^{k} D^{k}(t) h(t) .
$$

Здесь

$$
\begin{gathered}
B(t)=\lambda(t)-\int_{0}^{t} \dot{K}(t, s) \bullet d s \\
D(t)=B^{-1}(t) \frac{d}{d t}=\left(1-\int_{0}^{t} \frac{1}{\lambda(t)} \dot{K}(t, s) \bullet d s\right)^{-1} \frac{1}{\lambda(t)} \frac{d}{d t} ; \\
\left(\int_{t}^{s} B\left(s_{1}, \varepsilon\right) d s_{1}\right)^{k}=\int_{t}^{s} B\left(s_{1}\right) \int_{t}^{s_{1}} B\left(s_{2}\right) \cdots \int_{t}^{s_{k-1}} B\left(s_{k}\right) d s_{k} \ldots d s_{1} .
\end{gathered}
$$

Решение (18) запишется в виде

$$
u(t, \varepsilon)=\frac{1}{\varepsilon} \int_{0}^{t} U(t, s) \dot{h}(s) d s,
$$

где $U(t, s)$ - разрешающий оператор, удовлетворяющий уравнению

$$
\varepsilon \dot{U}(t, s)=B(t) U(t, s),\left.\quad U(t, s)\right|_{s=t}=I .
$$

Тогда верна цепочка равенств

$$
u(t, s)=\frac{1}{\varepsilon} \int_{0}^{t} U(t, s) \dot{h}(s) d s=\sum_{k=0}^{\infty} \frac{1}{\varepsilon} \int_{0}^{t} U(t, s) \frac{\partial}{\partial s}\left(\int_{t}^{s} B\left(s_{1}\right) d s_{1}\right)^{k} d s D^{k}(t) h(t) .
$$

Имеем

$$
\begin{aligned}
& \frac{1}{\varepsilon} \int_{0}^{t} U(t, s) \frac{\partial}{\partial s}\left(\int_{t}^{s} B\left(s_{1}\right) d s_{1}\right)^{k} d s D^{k}(t) h(t)= \\
& =-\left.U(t, s)\left(\int_{t}^{s} B\left(s_{1}\right) d s_{1}\right)^{k-1}\right|_{0} ^{t} D^{k}(t) h(t)+\int_{0}^{t} U(t, s) \frac{\partial}{\partial s}\left(\int_{t}^{s} B\left(s_{1}\right) d s_{1}\right)^{k-1} d s D^{k}(t) h(t)= \\
& =U(t, 0)\left(\int_{t}^{0} B\left(s_{1}\right) d s_{1}\right)^{k-1} D^{k}(t) h(t)+\int_{0}^{t} U(t, s) \frac{\partial}{\partial s}\left(\int_{t}^{s} B(s) d s\right)^{k-1} d s D^{k}(t) h(t)= \\
& =\ldots=U(t, 0) \sum_{m=0}^{k-1} \varepsilon^{k-1-m}\left(\int_{t}^{0} B(s) d s\right)^{k} D^{k}(t) h(t)-\varepsilon^{k-1} D^{k}(t) h(t) .
\end{aligned}
$$

Отсюда получим 


$$
\begin{aligned}
& u(t, \varepsilon)=U(t,0) \sum_{k=1}^{\infty} \sum_{m=0}^{k-1} \varepsilon^{k-1-m}\left(\int_{t}^{0} B(s) d s\right)^{m} D^{k}(t) h(t)-\sum_{k=1}^{\infty} \varepsilon^{k-1} D^{k}(t) h(t)= \\
&=\sum_{m=0}^{\infty} \varepsilon^{m} \sum_{k=0}^{\infty}\left(\int_{t}^{0} B(s) d s\right)^{k} D^{(k+(m+1))}(t) h(t)-\sum_{k=0}^{\infty} \varepsilon^{k} D^{(k+1)}(t) h(t)= \\
&=-\sum_{k=0}^{\infty} \varepsilon^{k} D^{(k+1)}(t) h(t) .
\end{aligned}
$$

Первое слагаемое равно нулю в силу условий (23) и (24):

$$
\sum_{k=0}^{\infty}\left(\int_{t}^{0} B(s) d s\right)^{k} D^{(k+(m+1))}(t) h(t)=D^{(m+1)}(0) h(0)=0
$$

что и требовалось доказать.

\section{СПИСОК ЛИТЕРАТУРЫ}

1. Филатов А. Н., Шершиков В. В. Асимптотические методы в атмосферных моделях. - Л.: Гидрометеоиздат, 1988.

2. Ломов С. А. Введение в общую теорию сингулярных возмущений. - М.: Наука, 1981.

3. Елисеев А. Г., Шапошникова Д. А. Задача инициализации сингулярно возмущенного интегрального уравнения Вольтерра второго рода с диагональным вырождением в случае $n \geqslant 3 / /$ Вестн. МЭИ. 2015. - 3. - C. $143-144$.

4. Ипатова В. М. Задачи инициализации для моделей общей циркуляции атмосферы// Тр. МФТИ. 2012. - 4, № 2. - C. 121-130.

Елисеев Александр Георгиевич

Национальный исследовательский университет «МЭИ», Москва

E-mail: eliseevag@mpei.ru

Ратникова Татьяна Анатольевна

Национальный исследовательский университет «МЭИ», Москва

E-mail: ratnikovata@mpei.ru

Шапошникова Дарья Алексеевна

Национальный исследовательский университет «МЭИ», Москва

E-mail: shaposhnikovda@mail.ru 